\title{
Replenished magma chambers: effects of compositional zonation and input rates
}

\author{
Herbert E. Huppert \\ Department of Applied Mathematics and Theoretical Physics, University of Cambridge, Silver Street, Cambridge, CB3 9 EW (England) \\ J. Stewart Turner \\ Research School of Earth Sciences, Australian National University, Canberra, A.C.T. 2600 (Australia) \\ and \\ R. Stephen J. Sparks \\ Department of Earth Sciences, University of Cambridge, Downing Site, Cambridge CB 2 3EW (England)
}

Received September 7, 1981

Revised version received November 19, 1981

\begin{abstract}
Several recent publications have reported investigations of the dynamical effects and geological consequences of the replenishment of a homogeneous magma chamber by the rapid injection of hot, relatively dense. fresh magma. The new magma forms a separate layer at the base of the chamber where it cools and crystallizes until the density of the residual liquid in the lower layer becomes equal to that of the upper layer, at which time the fluid in the two layers becomes intimately mixed. The fluid dynamical aspects of this model were confirmed by laboratory experiments using aqueous solutions. This paper reports three experimental variations on the basic theme which examine the effects of the rate of injection into homogeneous and compositionally stratified chambers. First, the rapid injection of a hot, dense layer beneath a fluid with a lower temperature and a vertical compositional gradient is considered. A series of layers gradually builds up from the base of the upper gradient region, while the lower layer cools, crystallizes and finally overturns. In this situation, however, the rise of the lower-layer fluid is restrained by the density gradient and mixing takes place with only part of the upper fluid. Second, the slow influx of a hot dense liquid beneath a cold, less dense homogeneous layer is considered. A considerable quantity of the input fluid cools and crystallizes immediately, releasing some lighter fluid to mix with the fluid in the homogeneous layer. With time, some dense input fluid spreads along the bottom so that locally the situation is the same as the one considered in the previous studies, with a local overturning occurring when the density of the lower fluid has decreased sufficiently. The deposition of crystals continues and some rather fine, chimney-like structures can develop which bear a resemblance to the "black smokers" recently discovered on the sea floor. Third, a combination of the first two variations is studied by the consideration of the slow influx of hot. dense liquid beneath a uniformly cooler fluid with a vertical compositional gradient. The input fluid is constrained in its rise by the density gradient. As the influx continues, the additional thermal energy is also confined and causes the crystals to redissolve and build up a lower layer, as was observed in the case of a rapid injection beneath a compositional gradient.
\end{abstract}

\section{Introduction}

Chemical concepts involved in the differentiation of magma in magma chambers have, over several decades, evolved into a sophisticated and complex body of knowledge. At the same time, the development of concepts on the important physical and dynamical processes involved in magma 
chambers has received much less attention. However, during the last few years some novel physical ideas, mainly involving the application of fluid dynamics, have been introduced. In particular, it has been recognised that the processes of crystallization and convection in magma chambers are likely to be much more complex than was formerly thought $[1,2]$.

A phenomenon of major petrological interest is the replenishment of a magma chamber by an influx of fresh magma from depth. A wealth of petrological, geological and physical evidence suggests that replenishment is an important process determining eruptive behaviour and some petrological and geochemical characteristics of volcanic rocks. It may also be responsible for the formation of certain kinds of compositional layering in plutonic rocks. Evidence for the replenishment of magma chambers and documentation of its petrological importance can be found scattered through the literature [3-7].

In a recent series of publications, we considered some of the fluid dynamic aspects of magma chamber replenishment and their petrological and volcanological implications $[4,5,7,8]$. These studies considered simple situations where a single influx of new fluid entered a chamber of uniform composition and temperature. Sparks et al. [4] discussed the situation of hot, light fluid entering a chamber containing colder, heavier fluid. Huppert and Sparks $[5,7]$ investigated the situation where hot, dense fluid is rapidly implaced beneath colder, lighter fluid. They concluded that the new fluid forms a separate layer at the base of the chamber, with vigorous convection in both layers driven by the transfer of heat across the relatively thin, non-turbulent interface separating the two layers. At the same time there is a negligibly small compositional flux across the interface. As the lower layer cools and crystallizes, the density of the residual liquid decreases and can reach the same density as the cooler fluid above, at which time the two fluids would begin to mix.

The model was applied by Huppert and Sparks to the petrogenesis of basaltic lavas and associated mafic and ultramafic plutonic rocks. They discussed how the model provides an explanation of why basalts erupted at mid-ocean ridges are re- stricted to liquid compositions with $\mathrm{MgO}<10.0 \%$. They also showed that it explains the formation of the cyclic layering which characterizes the ultramafic parts of cumulate rocks and the formation of quench textures in plutonic rocks.

The major predictions of the theoretical model were supported by a series of laboratory experiments conducted by Huppert and Turner [8] using aqueous solutions of $\mathrm{KNO}_{3}, \mathrm{NaNO}_{3}$ and $\mathrm{K}_{2} \mathrm{CO}_{3}$. The lower layer consisted of a hot $\mathrm{KNO}_{3}$ solution, for which the density of the saturated solution decreases as the temperature decreases and $\mathrm{KNO}_{3}$ crystallizes. Above this layer was a much deeper layer of colder, less dense solution of either $\mathrm{NaNO}_{3}$ or $\mathrm{K}_{2} \mathrm{CO}_{3}$. Huppert and Turner observed a strong convective transfer of heat across the interface between the two layers at a rate which they were able to predict quantitatively. As the lower layer cooled, $\mathrm{KNO}_{3}$ crystals grew mainly at the bottom while the density of the residual liquid decreased until it became equal to that of the upper layer. At this time the interface broke down and the two layers became intimately mixed with a stratum of $\mathrm{KNO}_{3}$ crystals remaining at the base. In general, the experimental investigations confirmed the feasibility of exploiting simple laboratory experiments to explore effects of convection and crystallization in analogous geological systems.

These previous studies considered possibly the simplest fluid dynamical situation of magma chamber replenishment, in which compositionally uniform, dense, hot fluid enters a chamber of compositionally uniform, cold fluid. There are, however, many other situations which can arise. In this paper we present a mainly experimental investigation of three other situations of magma chamber replenishment which could occur in nature. The first situation is the injection of hot, dense fluid into a chamber which is compositionally stratified. This case is discussed in most detail because of the range of geological applications which we can immediately identify. The second situation is the slow, continuous input of hot, dense fluid into a chamber filled with homogeneous, cold, less dense fluid. The third case is the slow, continuous input of hot, dense fluid at the base of a compositionally stratified chamber.

The geological justification for considering the 
effects of stratification is that compositionally and thermally zoned magma chambers are now known to occur in many volcano-tectonic settings [3,9-12]. Evidence for such zonation is widespread in volcanic rocks of intermediate to silicic composition both in calc-alkaline and tholeiitic provinces. In some cases, the changes in composition and temperature appear to be gradual $[9,11]$ and in others there are sharp discontinuities in composition [10]. Compositional gradients can arise naturally in magma chambers during crystallization by cooling through the containing walls or by melting of country rock $[10,13,14]$. Another way of generating compositional gradients is by repeated replenishment of a chamber.

All the experiments reported in this paper were conducted using the techniques described by Huppert and Turner [8] and further details are presented there. In particular, the laboratory chamber was a Perspex container measuring $40 \times 20 \times 30$ $\mathrm{cm}$ high and the input fluid was a solution of $\mathrm{KNO}_{3}$, whose saturation density as a function of temperature and concentration is graphed in Huppert and Turner [8, fig. 1].

In each section, after describing the experimental observations and related theory, we discuss some geological consequences of our findings. $\mathrm{Fi}$ nally, in the last section, we attempt to pull together the fluid dynamical and geological implications of the many variations.

\section{Rapid injection beneath a compositional gradient}

We carried out two virtually identical experiments in this case. In addition to the visual observations conducted during both experiments, we took colour photographs of the first, some of which are reproduced in black and white in Fig. 1, and made a cine film of the second. We will describe the first experiment in quantitative detail, but it should be remembered that the qualitative features described are appropriate for all such situations. The description incorporates much of what is already known from the similar situation of applying a constant heat flux to the base of a uniform salinity gradient $[15,16]$.

Solutions of $\mathrm{K}_{2} \mathrm{CO}_{3}$ at $16.6^{\circ} \mathrm{C}$ were fed into the experimental tank to a depth of $23.6 \mathrm{~cm}$ by the standard double-bucket method $[17,18]$ to produce a linear density gradient with a specific gravity of 1.281 at the bottom and 1.256 at the top. A 43.4 wt.\% $\mathrm{KNO}_{3}$ solution at $67.0^{\circ} \mathrm{C}$ with a specific gravity of 1.310 was injected in $2 \frac{1}{4}$ minutes beneath the $\mathrm{K}_{2} \mathrm{CO}_{3}$ gradient. The total depth added was $5.5 \mathrm{~cm}$.

Thermal convection in the lower layer was visible from the start. Heat was transferred to the upper, compositionally stratified solution and parcels of fluid began to rise. However, because of the slow molecular diffusion of $\mathrm{K}_{2} \mathrm{CO}_{3}$, the fluid parcels retained their chemical composition and, in contrast to the case of an homogeneous upper layer, were inhibited in their rise by the decreasing ambient density. Parcels rose to a height where the net density difference was zero (i.e., the density deficit due to temperature equalled the density excess due to composition) and formed a convecting layer. As more heat was transferred from the injected $\mathrm{KNO}_{3}$ layer, the thickness of the $\mathrm{K}_{2} \mathrm{CO}_{3}$ layer above it slowly increased. Simultaneously a thermal boundary layer developed above the convecting $\mathrm{K}_{2} \mathrm{CO}_{3}$ layer, and with time this boundary layer became unstable and initiated a second convecting layer in the $\mathrm{K}_{2} \mathrm{CO}_{3}$ gradient. This process was repeated to produce a third, fourth, ... convecting layer, as seen in Figs. 1 and 2. At times, the densities of two adjacent layers became equal and the interface between the layers broke down and the layers merged (Fig. $1 \mathrm{~b}$ and 1c). During the formation of the series of convecting layers, the $\mathrm{KNO}_{3}$ solution cooled, crystallized and the density of the residual solution decreased. After a time this density became equal to that in the lowest $\mathrm{K}_{2} \mathrm{CO}_{3}$ layer and the interface between the two layers broke down. As described in the previous experiments [8], the $\mathrm{KNO}_{3}$ solution rose and mixed with the fluid above it, but in contrast to the homogeneous situation, the rise was inhibited by the density variation in the $\mathrm{K}_{2} \mathrm{CO}_{3}$, and the $\mathrm{KNO}_{3}$ mixed only with the lowest $\mathrm{K}_{2} \mathrm{CO}_{3}$ layer (Fig. 1d and le). The newly formed lowest layer was still hotter than the fluid above and some additional convecting layers developed. After some time the fluid became isothermal, all convection ceased and a series of compositional layers remained with a 

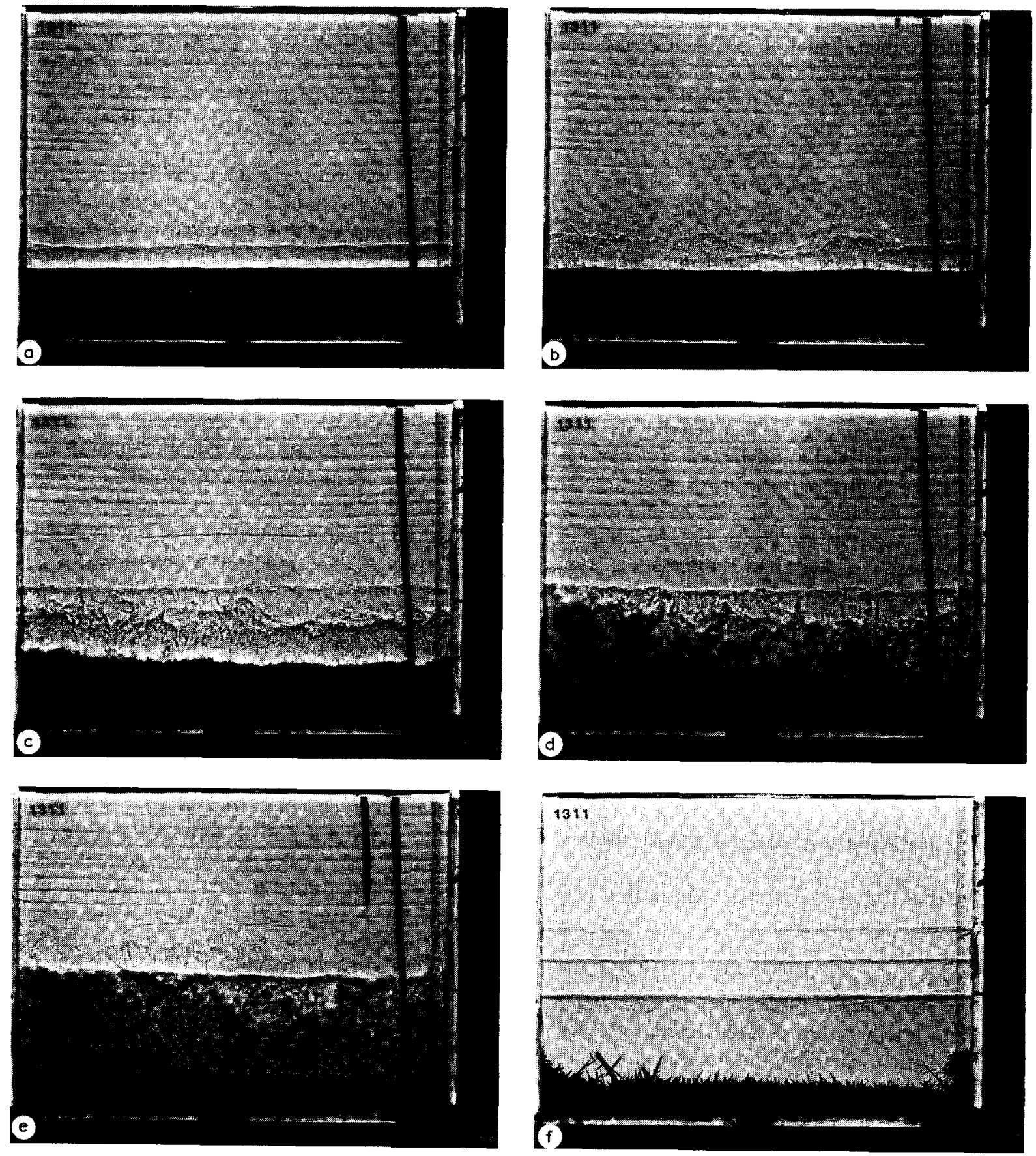

Fig. 1. Shadowgraph views of a rapid injection of hot, dyed $\mathrm{KNO}_{3}$ solution beneath a $\mathrm{K}_{2} \mathrm{CO}_{3}$ compositional gradient. The clock shows the time in hours, minutes and seconds since the commencement of the injection. Twenty-four hours should be added to the clock in the last view. 


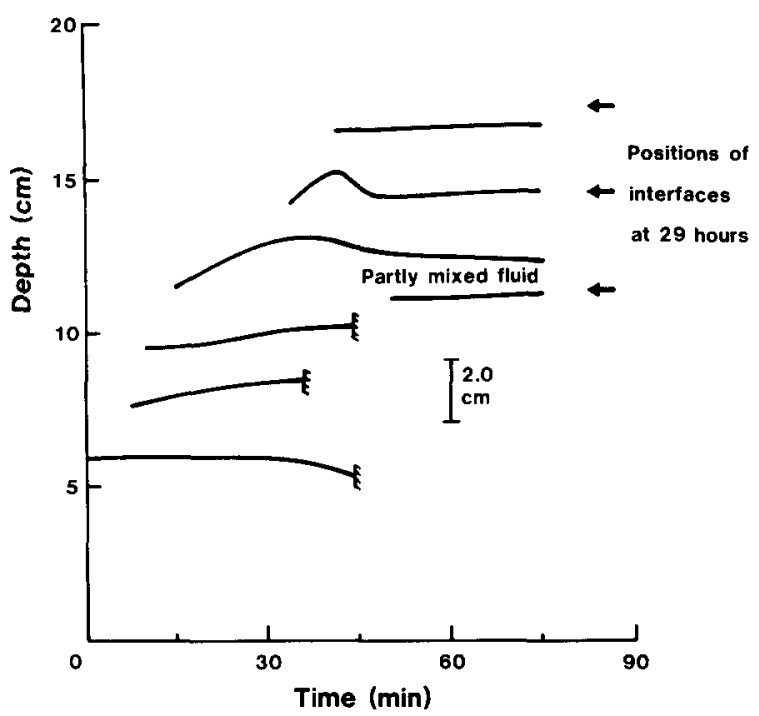

Fig. 2. The depths of the interfaces observed in the experiment of Fig. 1 as a function of time. Smooth curves have been drawn through the individual observations, which represent a visual average over the width of the tank. The short vertical lines with hatching to the right indicate that there was no interface remaining at that depth at that time. The new interface appearing at the top of the deep lower layer after 50 minutes bounds partly mixed fluid which separated out at that time.

crystal stratum at the base (Fig. 1f). During the next five days no change occurred; in particular, the crystals neither grew nor dissolved.

Variation of the external parameters - the depth of the lower layer, the density gradient, the temperature differences between the two fluids and the difference in densities between the lower layer and the fluid just above it - would be expected to alter the number of convecting layers, their thickness, their times of formation and the times at which overturning occurs. Explicit numerical predictions for aqueous solutions could be obtained by modifying the programme of Huppert and Linden [16], for uniformly heating a salinity gradient, to accommodate the presence of an insulated base. The modification requires merely that the term on the right-hand side of (3.3) in Huppert and Linden [16], representing the heat flux through the base of the container, be set equal to zero. Two statements can be made, however, without detailed calculations.

First, the cooling of the $\mathrm{KNO}_{3}$ layer takes place more slowly and the time for overturning is increased in the presence of a compositional gradient above it. This is because the heat lost from the $\mathrm{KNO}_{3}$ layer is distributed over a small height and the temperature just above the $\mathrm{KNO}_{3}$ layer is thus increased. Hence, the temperature difference across this lowest interface is decreased, which results in a slower heat flux and a smaller rate of cooling. Second, Huppert and Linden's calculations and experiments showed that the thickness of the convecting layers in a series (aside from the lowest one) tends to be uniform and thus the mean thickness, $\bar{H}$, is independent of the heat flux, which varies from layer to layer. Dimensional analysis then shows that

$$
\bar{H} \propto \kappa_{T}^{1 / 2}(g \mathrm{~d} \rho / \mathrm{d} z)^{-1 / 4},
$$

where $\kappa_{T}$ is the molecular diffusivity of heat, $g$ is the acceleration due to gravity, $\mathrm{d} \rho / \mathrm{d} z$ is the vertical density gradient and for $\mathrm{NaCl}$ in water the constant of proportionality is 51 . Using values for the experiment just described, we find that $\bar{H}=2.0$ $\mathrm{cm}$, in good agreement with the thickness of layers as shown in Fig. 2. Note that the constant of proportionality is in general a function of the thermal and compositional diffusivities and the viscosity of the fluid and, while it is approximately the same for all solutes in aqueous experiments, the value of 51 is not appropriate for magmas.

Finally, we present a brief explanation of the thin layers visible in the upper part of the $\mathrm{K}_{2} \mathrm{CO}_{3}$ solution in Fig. la-e. They are due to the difference in temperature between the tank walls, at room temperature $\approx 21^{\circ} \mathrm{C}$, and the fluid $\approx 17^{\circ} \mathrm{C}$. The relatively hot wall causes neighbouring fluid to rise. But the rise is again constrained by the density gradient and a series of layers results. The most extensive investigation and discussion of this phenomenon to date appears in Huppert and Turner [19], who determined that the layer scale, $h$, is given by:

$h=0.65\left(\rho_{\mathrm{W}}-\rho_{\mathrm{I}}\right) / \frac{\mathrm{d} \rho}{\mathrm{d} z}$,

where $\rho$ is the density of the fluid and the subscripts "W" and "I" indicate values at the wall and in the far interior of the fluid, respectively. Using values appropriate to the experiment described, we 
find that $h=1.2 \mathrm{~cm}$, in good agreement with the layer scales seen in Fig. 1. It should be noted that the constant of proportionality in (2) may be a function of the ratio of the molecular diffusivities of heat and composition (in particular, it may be different for magmas), and that the form of (2) is invalid if the fluid is crystallizing and releasing fluid of different density.

In a compositionally zoned magma chamber replenished by hot, primitive magma, the experiment together with theory [16] suggest that the thermal disturbance can initially be transmitted into the compositionally stratified part of the chamber although there is no immediate mixing of the new and old magmas. The new layer of hot magma acts as a source of heat which breaks down any initially smooth density gradient into a series of layers. The transfer of heat from the new magma will occur by convection within these layers, which will be very much more rapid than any conductive process. The composition and temperature of each layer will be spatially uniform and fluid from neighbouring levels in the magma chamber will be thus mixed together during the formation of layers.

The mixing and layering produced by influx of hot, dense magma will have consequences in the petrological features of eruptive volcanism. For example, the heating event associated with a substantial replenishment could lead initially to resorption of phenocrysts. The mixing events caused by merging or formation of layers would bring phenocrysts from each layer into a new thermal and compositional environment with which they would try to re-equilibrate. The turbulence induced during mixing would produce smallscale fluctuations in temperature and melt composition. Features such as oscillatory zoning in plagioclase feldspars could result for example. However, only relatively small compositional variations can be produced by this mechanism, because the process involves mixing of fluids from neighbouring levels which are close in composition and temperature.

In the experiment, mixing of fluids of contrasting composition and temperature occurred when crystallization in the lower layer produced a residual fluid which reached the same density as the lower parts of the compositionally stratified fluid.
A physically analogous situation can arise in magma chambers in the tholeiitic association where, in the compositional range of basalts, more differentiated magmas are often denser than more primitive magmas $[4,20]$. Tholeiitic magmas more differentiated than ferrobasalt, however, become progressively lower in density with differentiation and can form compositionally stratified systems $[11,12]$. The replenishment of a magma chamber zoned from rhyolite at the top to basalt at the base can now be considered. If picritic basalt enters the base of the chamber a separate layer can form beneath the basaltic lower part of the chamber, in a manner similar to that outlined by Huppert and Sparks [5,7]. Crystallization of olivine in the picritic basalt layer can produce low-density residual basaltic magma $[4,20]$ which can mix with the overlying differentiated basaltic part of the chamber, but mixing will be limited to the lower parts of the chamber due to the strong compositional stratification.

\section{Slow, continuous input beneath a homogeneous layer}

The theoretical model of Huppert and Sparks $[5,7]$ assumes that the injection of the lower-layer fluid is "instantaneous", or explicitly that the injection takes place in a time which is short compared with the time scale describing the cooling of the lower layer. They considered their assumption of episodic, instantaneous inputs to be justified by the episodic rapid outputs observed in many volcanic environments and the abrupt compositional changes observed in cyclic layering in ultrabasic intrusions. However, it is feasible that in some cases the input is slow and continuous and the experiments to be described in this section were carried out with this possibility in mind.

We carried out eight experiments, taking photographs during all of them and making cine films of four. For each experiment the homogeneous layer was nominally $20 \mathrm{~cm}$ deep and consisted of 38 wt.\% $\mathrm{NaNO}_{3}$ (specific gravity 1.31) at an initial temperature of $10^{\circ} \mathrm{C}$, except for one experiment which used $\mathrm{K}_{2} \mathrm{CO}_{3}$. The input was nominally 46 wt.\% $\mathrm{KNO}_{3}$ (specific gravity 1.33) at an initial 
temperature of $65^{\circ} \mathrm{C}$. The $\mathrm{KNO}_{3}$ solution was input by a peristaltic pump at a rate which varied from experiment to experiment between 0.73 and $3.83 \mathrm{~cm}^{3} \mathrm{~s}^{-1}$.

In each experiment, as the input fluid was introduced it crystallized at the base of the container due to being rapidly quenched by the cold surrounding $\mathrm{NaNO}_{3}$ and a plume of depleted $\mathrm{KNO}_{3}$ solution rose to the surface (Figs. 3a and 4a). Except for the experiment carried out at the fastest input rate, which will be discussed separately below, crystallization to form an irregular amorphous layer on the container floor continued for quite some time. The input fluid seemed to flow in thin channels in the amorphous crystal mass and slowly extended the crystal/liquid boundary. Initially the shape of the crystal boundary was unconstrained by the container walls, but after a while a layer of loosely compacted crystals spread across the chamber floor and then filled the bottom of the container (Fig. $4 \mathrm{~b}$ and $\mathrm{c}$ ). During the influx relatively light, residual fluid produced by crystallization continually rose in a series of plumes and mixed with the original homogeneous fluid, and the $\mathrm{KNO}_{3}$ concentration of the fluid in the container thus increased. An indication of this is given by the steady darkening of the background in Figs. 3 and 4.

At a later time hot $\mathrm{KNO}_{3}$ solution trapped within the crystal pile broke through and fluid was seen to pond at the base of the homogeneous layer. This was first noticed after 15 minutes in the experiment depicted in Fig. 3 and after 1 hour 31 minutes in that depicted in Fig. 4. Fluid continued to flow out of the crystal pile at a number of exit points and the layer of hot dense $\mathrm{KNO}_{3}$ solution, as evident. in Fig. 3b, increased in depth and crystallized with the long dendritic morphology characteristic of the experiments with rapid injection beneath a homogeneous layer (cf. [8, fig. 5]). Often some of the crystals in contact with the main fluid layer began to redissolve slowly at this stage. Due to the history of crystallization and subsequent formation of the lower layer, it was frequently stratified and compositional convection associated with the cooling was restricted to the upper parts of this layer. Nevertheless, in a num- ber of experiments the density of the lower layer became sufficiently low that the interface broke down locally and an overturn occurred, as is evident in Fig. 3c. The crystal pile remained relatively flat-topped during this time, as is most clearly seen in Figs. $3 c$ and $4 b, c$.

In three of the experiments, after some 2 hours many of the exit points became blocked and a "chimney" began to grow directly above the input, as is seen in Figs. 3d, e and 4d. Hot $\mathrm{KNO}_{3}$ solution flowed up the interior of the "chimney" and some of the fluid crystallized at the top and thus increased the height of the "chimney". Later examination of the "chimney" which grew in the experiment depicted in Fig. 4 indicated that it had a rather complex internal structure, with a cluster of thin-walled conduits leading from the base to the top, thus allowing for vertical flow of fluid. Two photographs of this structure, taken after the tank was drained, are reproduced in Fig. 5. The top of the "chimney" remained nearly horizontal as it grew, indicating that denser fluid was being forced up to that level and ponding there, causing further crystal growth as it flowed over the top and down the sides. This contributed to the fluid layer at the foot, as is most clearly shown in Fig. 3e. After a time the "chimney" developed holes in the side and at the base where fluid was able to flow out. If the input was stopped and the tank left undrained, the "chimney" slowly dissolved (Fig. 3f), and the dense solution flowed down and produced a stratified layer on the bottom. We cannot be certain about what originated these "chimneys". In two experiments they begin to grow minutes after we drained the tank of some $10 \mathrm{~cm}$ of fluid because it was in danger of overflowing. The reduced head may have facilitated the vertical motion of the input fluid. In another experiment, however, the growth began just after the peristaltic pumping rate was inadvertently reduced by almost a factor of 3 .

In the experiment at the most rapid input rate, the initial amount of crystallization was rather small. With continuing input of hot dense fluid the crystals redissolved and the fluid formed a lower layer of ever increasing height. When the layer was $11 \mathrm{~cm}$ deep the input was stopped, and, while crystals continued to grow for some time, the 

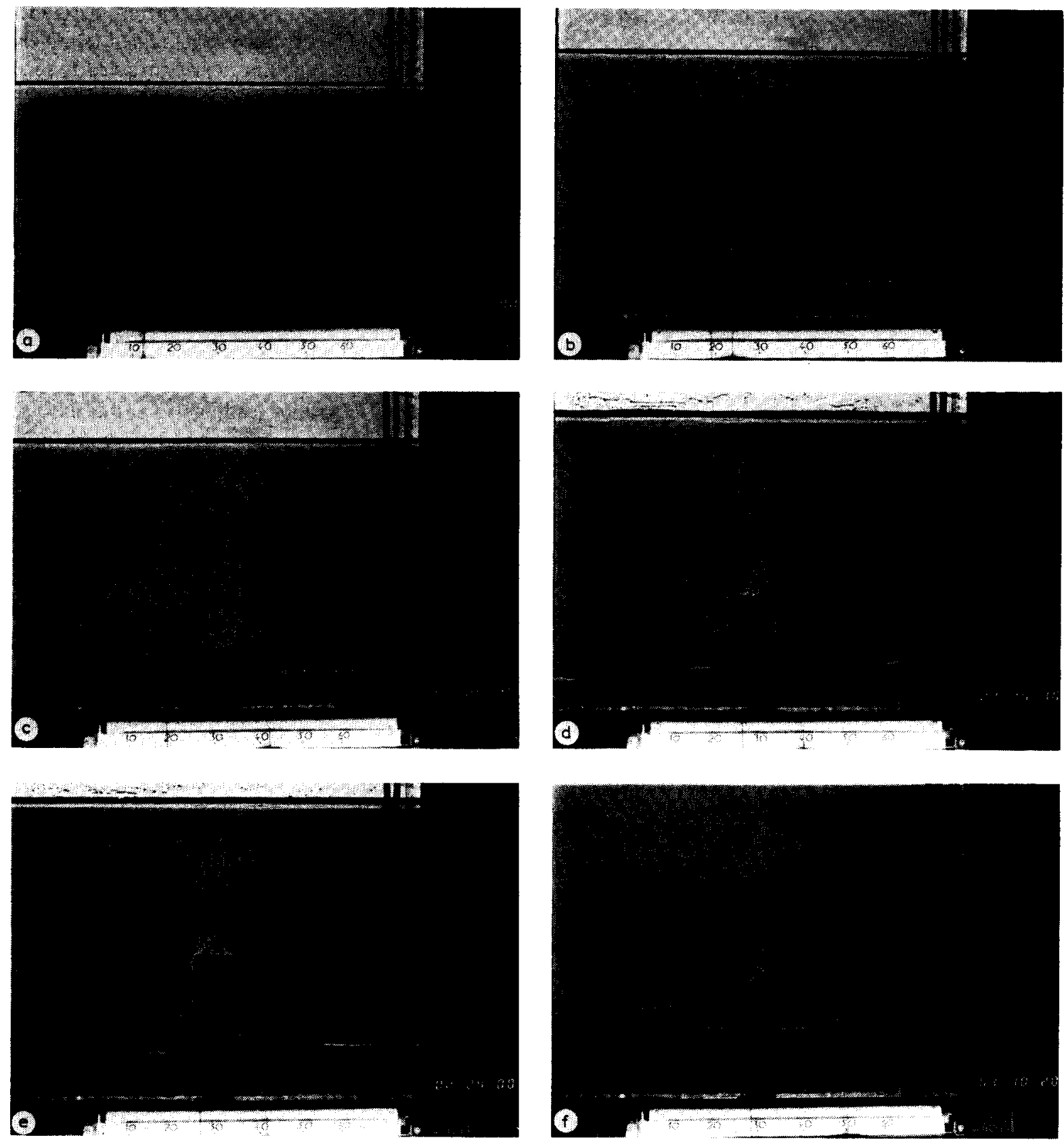

Fig. 3. Shadowgraph views of a slow, continuous input of hot, dyed $\mathrm{KNO}_{3}$ solution beneath a homogeneous layer of $\mathrm{NaNO}_{3}$ solution. The chart recorder at the bottom displays the temperatures measured by thermistors at the bottom of the two probes at the right-hand end of the tank. The initial homogeneous layer properties were: depth $20.1 \mathrm{~cm}$; concentration 38 wt. \%; specific gravity 1.313 ; and temperature $8.5^{\circ} \mathrm{C}$. The input properties were: input rate $0.85 \mathrm{~cm}^{3} \mathrm{~s}^{-1}$; concentration $46 \mathrm{wt} . \%$; specific gravity 1.335 ; and temperature $60^{\circ} \mathrm{C}$. The input ceased at $02: 59: 15$. 

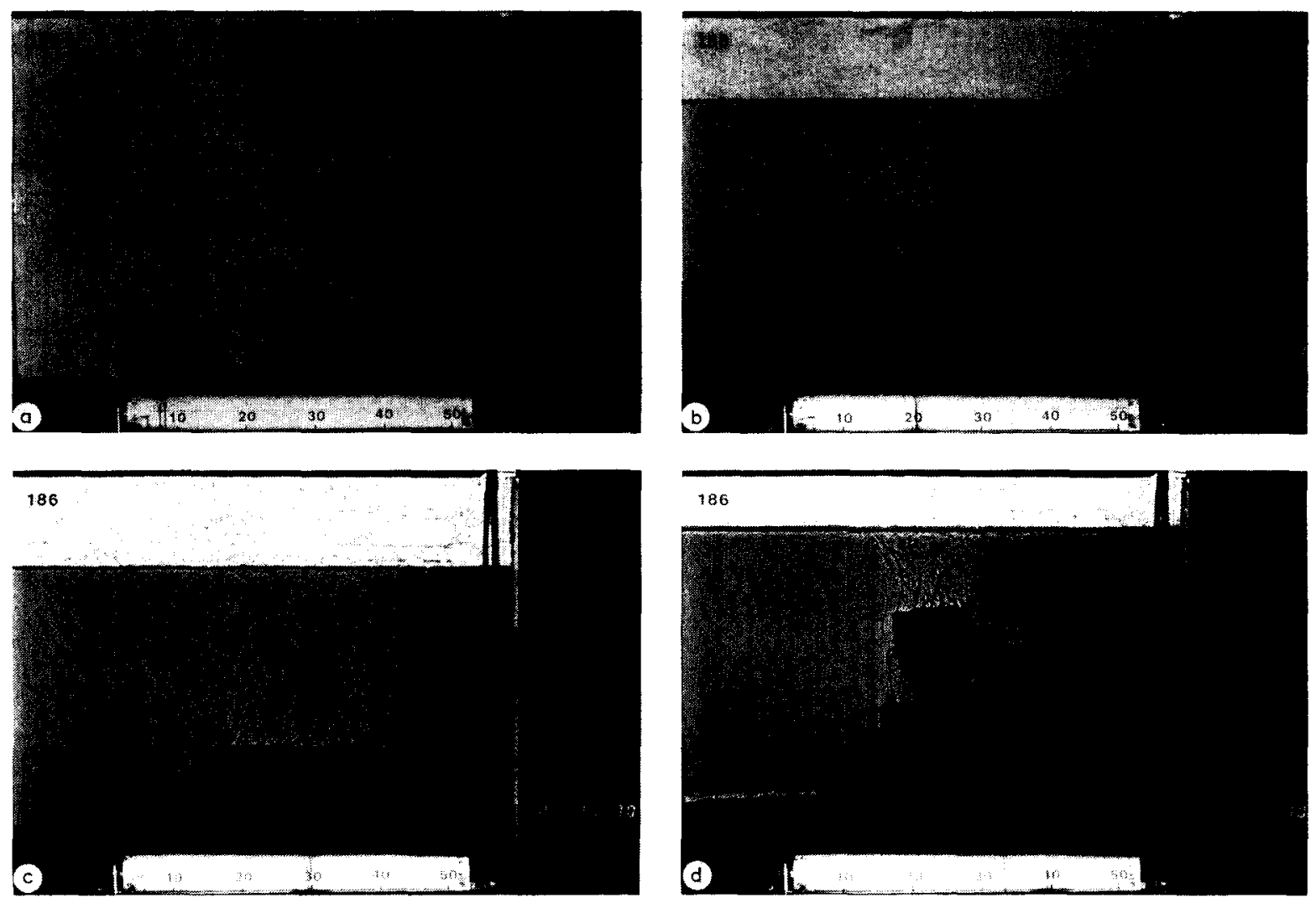

Fig. 4. Shadowgraph views of a slow. continuous input of hot, dyed $\mathrm{KNO}_{3}$ solution beneath a homogeneous layer of $\mathrm{K}_{2} \mathrm{CO}_{3}$ solution. The initial homogeneous layer properties were: depth $18.0 \mathrm{~cm}$; specific gravity 1.318 : and temperature $8.2^{\circ} \mathrm{C}$. The input properties were: input rate $1.02 \mathrm{~cm}^{3} \mathrm{~s}^{-1}$; specific gravity 1.338: and temperature $66^{\circ} \mathrm{C}$. The input ceased at 2:58:00

density of the residual liquid did not decrease sufficiently for the layer to overturn. We did not attempt to determine, even roughly, the input rate at which, as far as the subsequent fluid dynamical development is concerned, the injection is effectively instantaneous. The value would depend quite critically on the density and temperature of the two fluids and the depth of the upper layer.

In general, explicit details of the effects noticed in this series of experiments were rather sensitive to the input rate. This implies that it would be difficult to make quantitative predictions for particular situations of, for example, the size and shape of the crystal pile and the temperature and composition of the surrounding fluid.

The most important geological implication of these experiments is that even with a slow, con- tinuous input, a magma chamber can act as an effective fluid dynamical buffer between the input and output. For example, a slow, continuous input of hot dense picritic basalt would induce the release of fluid of relatively lower $\mathrm{MgO}$ content, and hence less dense, and leave a quenched olivine pile at the base of the chamber. In previous studies $[5,7]$ it was demonstrated that a sudden influx of hot picritic basalt into a mid-ocean ridge magma chamber would restrict the composition of erupted lavas to basalts with $\mathrm{MgO}<10 \%$. The current experiments indicate that the same fluid dynamic restrictions probably occur even if the input is slow and continuous. In the experiment the layer of crystals spread over the chamber floor, limited only by the sides of the container. Evidently an extensive layer of crystals can form even when the 

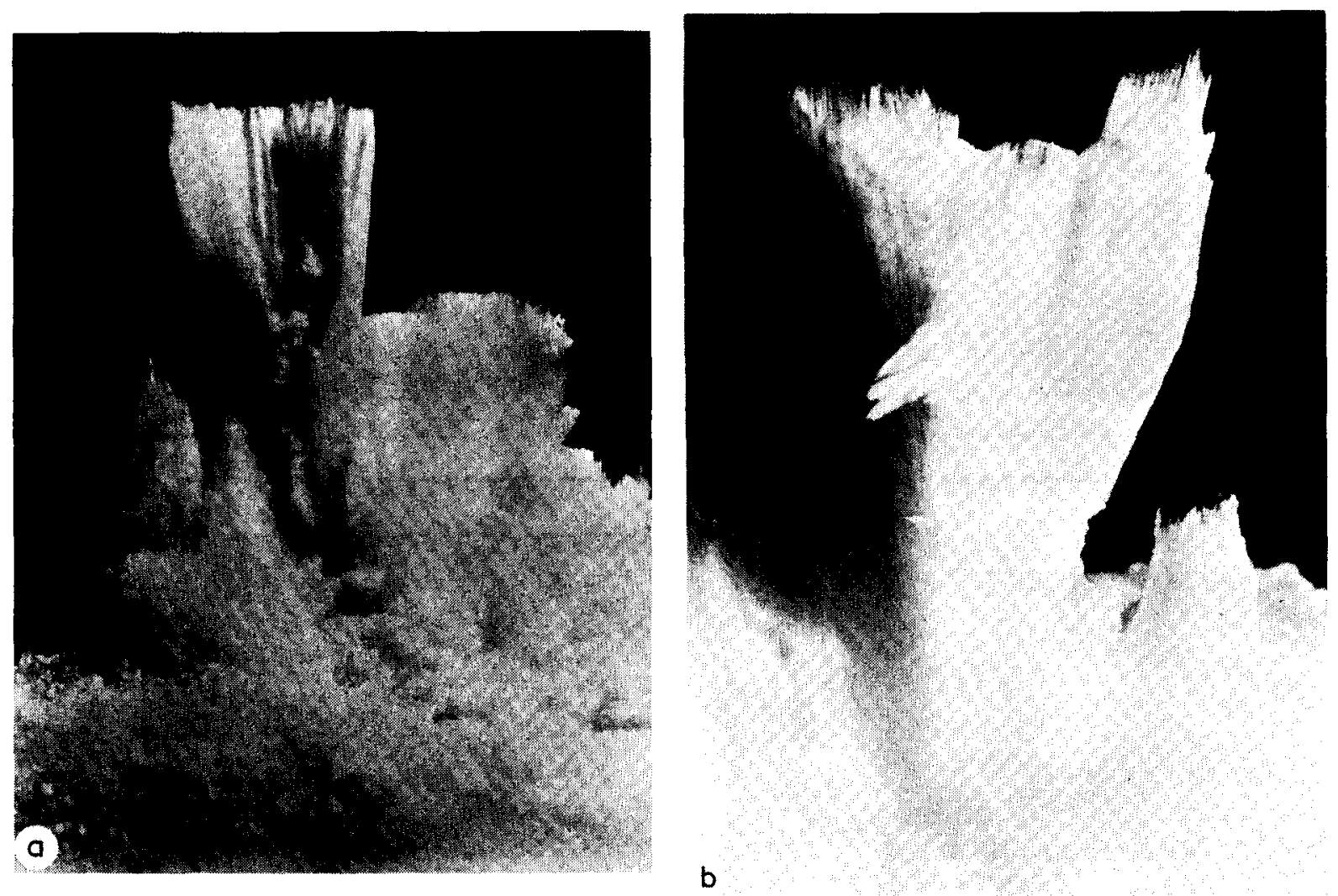

Fig. 5. Two views of the "chimneys" produced in the experiment described in Fig. 4. These structures formed after crystallization had blocked the outlets in the lower layer and hot fluid was forced upward through a cluster of thin-walled conduits. The nearly horizontal tops indicate that the denser fluid ponded at that level and was responsible for further crystal growth as it flowed over the edge and down the sides.

input is slow. An interesting feature of the experiment is that some dense input fluid eventually does form a separate layer covering previously formed crystals. This layer itself cools, crystallizes and eventually overturns. This shows that even with a continuous inflow at source, cyclic episodes of crystallization and overturning could occur on a magma chamber floor.

Finally, it is tempting to identify the "chimneys" with the "smokers" recently discovered on the East Pacific Rise and the Galapagos Ridge $[21,22]$. The experiments just described suggest how these "smokers" could have been built up by the quenching of hot, metal-rich solutions as they emerged from the sea floor into cold seawater. Our results indicate, however, that such deposits must have grown from the outside, with newly emergent solution cooling and crystallizing successively around the top of the growing column. Internal crystallization, on the other hand, would quickly block the vents. The direct application of our results implies that the hot solutions forming the smokers were either denser than seawater when they emerged, or became denser due to nonlinear mixing effects [14], and then flowed over the top and down the sides of the growing columns. It is possible, however, that processes we have not modelled, such as the formation of precipitates due to chemical reactions, or the leaking of fluid through porous walls, could play a significant role in the growth of smokers. 

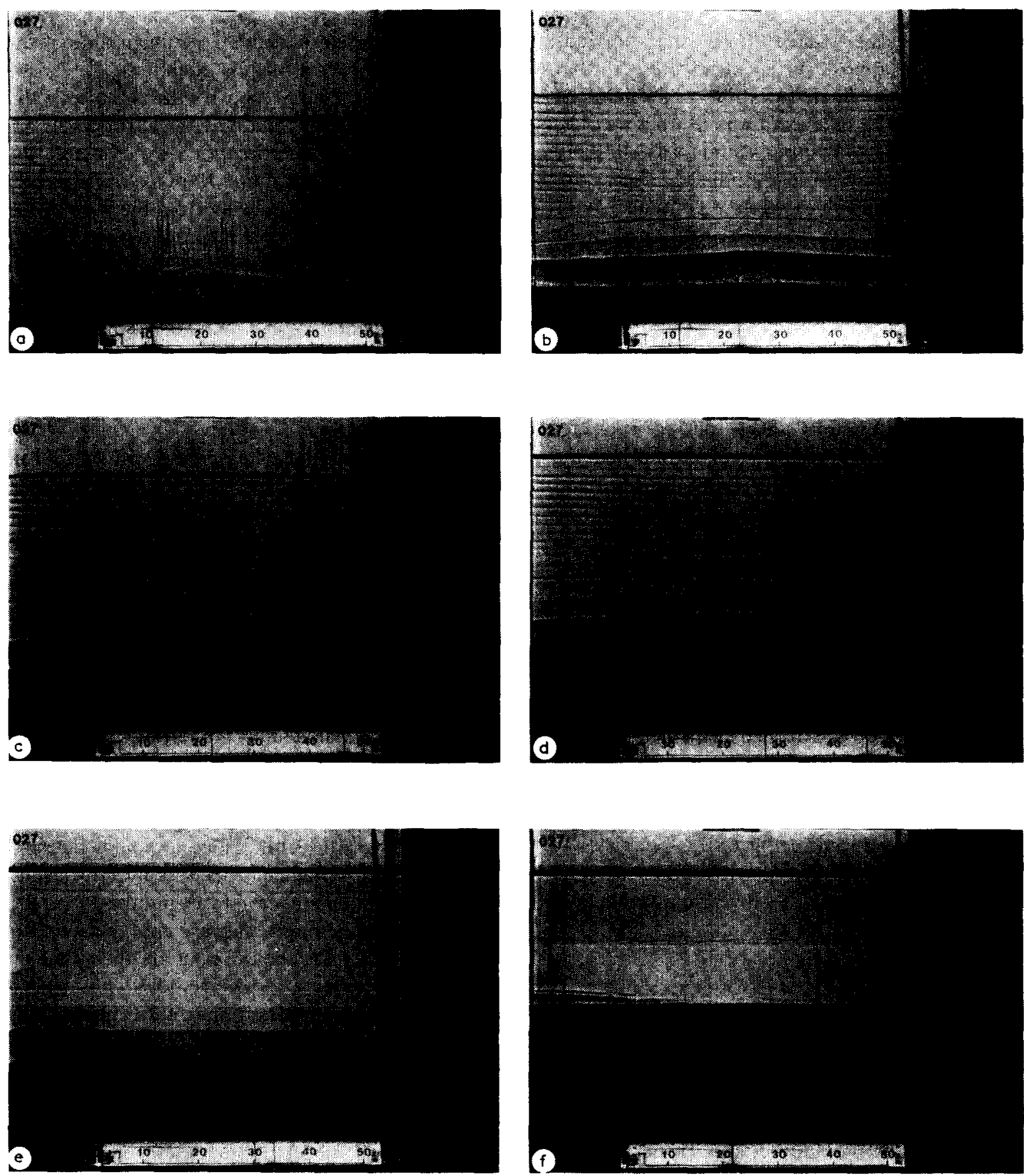

Fig. 6. Shadowgraph views of a slow, continuous input of hot, dyed $\mathrm{KNO}_{3}$ solution beneath a compositional gradient. The initial upper solution properties were: depth $18.3 \mathrm{~cm}$; specific gravity at bottom 1.232 ; specific gravity at top $1.202 ;$ and temperature $11.9^{\circ} \mathrm{C}$. The initial input properties were: input rate $1.12 \mathrm{~cm}^{3} \mathrm{~s}^{-1}$; specific gravity 1.254 : and temperature $61^{\circ} \mathrm{C}$. The input ceased at $1: 25: 00$. 


\section{Slow, continuous input beneath a compositional gradient}

The knowledge gained from the previous experiments is almost sufficient to predict what occurred in this final variation in which the observed sequence of events was as follows. The hot, slowly injected input fluid was initially quenched, and much of it crystallized. The warm, relatively light fluid released as a consequence of the crystallization was, however, restrained in its rise by the compositional gradient. This limitation resulted in the formation of a lower layer which enveloped the crystals and the input, though a few thermal plumes initially extended above the lower layer. The crystals and the enveloping layer as well as the plumes are clearly seen in Fig. 6a, which reproduces photographs taken during such an experiment. The enveloping layer diminished the cooling effect of the cold fluid above the input and crystallization took place less rapidly than if the input had been under a homogeneous layer. The initially formed crystals can even redissolve as the temperature of the enveloping layer increases. At the same time the base of the compositional gradient evolved into a series of convecting layers, in a manner analogous to that already described in section 2 (Figs. 6b, c, d).

When the input was turned off, further evolution was similar so that for a rapid injection beneath a compositional gradient. Though sudden overturning of the lower layer did not occur in the experiment reproduced in Fig. 6, this layer slowly grew deeper in time as less dense fluid released by the crystallization eroded the overlying stable density gradient (Figs. 6e, f).

\section{Conclusions}

There are a number of unifying features associated with the variety of different phenomena displayed by the current experiments and those reported previously by Huppert and Turner [8]. The incoming hot fluid, being denser than the resident fluid, is initially confined to the base of the chamber (except for a small amount of light fluid released following crystallization when there is a slow input beneath a homogeneous layer, as described in section 3). The input thermal energy initiates high Rayleigh number turbulent convection, which, because of the large magnitude of the Rayleigh number in magma chambers [5], can be anticipated there also. As a result of the crystallization and different fluid dynamical processes operating in each of the described experiments, the concentration of the input fluid is considerably reduced before it moves away from the base of the chamber. A chamber can thus act as an efficient buffer between the composition of input and output magma. Finally, in those cases where the resident fluid is compositionally stratified, the induced convection can break down an initially smooth concentration and density gradient into a series of layers with uniform concentration and density in each layer.

We hope that the experiments will act as an incentive for petrologists to investigate magma chambers with these new fluid dynamical concepts in mind. It should be remembered, however, that some of the details of the fluid dynamical evolution of the input fluid in the current experiments are rather sensitive to external parameters, such as flow rates, temperature and density differences. It should also be borne in mind that other fundamental fluid dynamical processes will be important in magma chambers. Examples include the rapid or slow influx of hot, compositionally rich or poor, relatively light fluid into a homogeneous or stratified fluid [4] and the cooling of a single layer from the side $[10,13,14]$. Thus, although the simplest and most basic concepts of convective evolution in magma chambers are beginning to be understood, and strongly suggest that petrologists and field geologists should examine their data in line with these new ideas, it should not be expected that all important magmatic phenomena can be described in detail by the fluid dynamical models investigated so far. Ideally, an interaction will be set up between petrologists, field geologists and fluid dynamicists which will lead to the development of more complete and quantitative models of magma chambers. 


\section{Acknowledgements}

Extremely capable technical assistance with the experiments was given us by Derek Corrigan and Ross Wylde-Browne, to whom we are grateful. Most of the experiments were conducted while H.E.H. was a Visiting Fellow at the Research School of Earth Sciences, A.N.U. He is grateful for many stimulating discussions with scientists at the School and for the financial support of the A.N.U. and The Royal Society, which made the visit possible. Helpful comments on an earlier draft of the paper were made by C.H. Donaldson, A.R. McBirney and B.D. Marsh. This paper is Department of Earth Sciences contribution 175.

\section{References}

I A.R. McBirney and R.M. Noyes, Crystallization and layering in the Skaergaard intrusion, J. Petrol. 20 (1979) 487-554.

2 H.E. Huppert and J.S. Turner, Double-diffusive convection, J. Fluid Mcch. 106 (1981) 299-329. This review article devotes one section to geological applications and references many of the papers discussing the new ideas on magma chambers.

3 R.L. Smith, Ash-flow magmatism, Geol. Soc. Am. Spec. Paper 180 (1979) 5-28.

4 R.S.J. Sparks, P. Meyer and H. Sigurdsson, Density variation amongst mid-ocean ridge basalts: implications for magma mixing and the scarcity of primitive lavas, Earth Planet. Sci. Lett. 46 (1980) 419-430.

5 H.E. Huppert and R.S.J. Sparks, Restrictions on the compositions of mid-ocean ridge basalts: a fluid dynamical investigation, Nature 286 (1980) 46-48

6 M.J. O'Hara and R.E. Mathews, Geochemical evolution in an advancing, periodically replenished, periodically trapped, continuously fractionated magma chamber, J. Geol. Soc. London 138 (1981) 237-278.

7 H.E. Huppert and R.S.J. Sparks, The fluid dynamics of a basaltic magma chamber replenished by influx of hot, dense ultrabasic magma, Contrib. Mineral. Petrol. 75 (1980) 279 289

8 H.E. Huppert and J.S. Turner, A laboratory model of a replenished magma chamber, Earth and Planet. Sci. Lett. 54 (1981) 144-152.

9 W.S. Hildreth. The Bishop Tuff: evidence for the origin of compositional zonation in silicic magma chambers. Geol. Soc. Am. Spec. Paper 180 (1979) 43-72.

10 A.R. McBirney, Mixing and unmixing of magmas, J. Volcanol. Geotherm. Res. 7 (1980) 357-371.

11 G. Larsen and S. Thoraninsson. $\mathrm{H}_{4}$ and other acid Hekla tephra layers, Jökull 27 (1977) 28-46.

12 H. Sigurdsson and R.S.J. Sparks. Petrology of rhyolitic and mixed magma ejecta from the 1875 eruption of Askja. Iceland, J. Petrol. 22 (1981) 41-81.

13 J.S. Turner. A fluid dynamical model of differentiation and layering in magma chambers, Nature 285 (1980) 213-215.

14 J.S. Turner and L.B. Gustafson, Fluid motions and compositional gradients produced by crystallization or melting at vertical boundaries, J. Volcanol. Geotherm. Res. II (1981) 93-125.

15 J.S. Turner, The behaviour of a stable salinity gradient heated from below, J. Fluid Mech. 33 (1968) 183-200.

16 H.E. Huppert and P.F. Linden, On heating a stable salinity gradient from below, J. Fluid Mech. 95 (1979) 431-464.

17 G. Oster and M. Yamamoto, Density gradient techniques, Chem. Rev. 63 (1963) 257-268.

18 G. Oster, Density gradients, Sci. Am. 213 (1965) 70-76.

19 H.E. Huppert and J.S. Turner, Ice blocks melting into a salinity gradient, J. Fluid Mech. 100 (1980) 367-384.

20 E. Stolper and D. Walker, Melt density and the average composition of basalt, Contrib. Mineral. Petrol. 74 (1980) $7-12$

21 J.M. Edmond, Ridge crest hot springs: the story so far, EOS $61(1980) 128-131$

22 K.C. Macdonald and B.P. Luyendyk. The crest of the East Pacific Rise. Sci. Am. 255 (1981) 100-116 